\title{
A PRELIMINARY INVESTIGATION FOR THE USE OF DIGITAL GAMMA-GAMMA COINCIDENCE SPECTROMETRY TO DETECT ${ }^{239} \mathrm{Pu}$
}

\author{
Conny Egozi ${ }^{*}$, Francis Martinez ${ }^{2}$, Brandon De Luna $^{1}$, James Terry $^{1}$, Sheldon Landsberger
}

${ }^{1}$ University of Texas at Austin, Nuclear Engineering Teaching Laboratory, Pickle Research Campus, Austin, Texas, USA

${ }^{2}$ Los Alamos National Laboratory, Los Alamos, New Mexico, USA

\begin{abstract}
Gamma-ray spectrometry is one of the most effective ways to determine the activity of $239 \mathrm{Pu}$, depending on activity levels. However, often high backgrounds in complex spectra with low amounts of 239 Pu can increase detection limits. The effectiveness of the use of gamma-gamma spectrometry in the characterization of $239 \mathrm{Pu}$ is studied for the first time. Using the XIA Pixie-16 digital pulse processor, gamma-gamma coincidences were measured to study the unshielded background radiation as a function of the source to detector distance, and gating of several gamma-rays from ${ }^{239 P u}$. The Poisson distribution of the acquired pulses has been also verified. In addition, measurements demonstrated that the background radiation is strongly reduced, meaning gamma-gamma spectroscopy can be very effective to avoid the usual lead shielding. Other measurements were taken to assure Poisson statistics were attained in the digital system. Preliminary measurements revealed 3 orders of magnitude background reduction for the measurement of $239 \mathrm{Pu}$ using gated gamma-rays.
\end{abstract}

Key words: Background reduction, gamma-gamma coincidence spectrometry, Pixie XIA, Poisson statistics, ${ }^{239 P u}$ detection

\section{INTRODUCTION}

Gamma-ray spectrometry with a single germanium detector has been previously used for the characterization of the low-energy gamma rays of $239 \mathrm{Pu}$ [1]. In addition, it has been used for the determination of ${ }^{239} \mathrm{Pu} /{ }^{241} \mathrm{Pu}$ ratios in uranium-plutonium mixed oxides (MOX) [2]. However, the background when using a single unshielded gamma-ray detector can be on the order of thousands of counts, depending on the counting times, energy in the spectrum and detector efficiency causing increased detection limits. One method to reduce the background, and therefore the detection limits, is the use of both active and passive shielding. The passive shield involves the use of very low activity lead, and the active shield is comprised of a plastic scintillation plate [3]. Another option is the use of gamma-gamma coincidence, which has been used for ${ }^{134 \mathrm{Cs}}$ [4], among other isotopes, but not for ${ }^{239} \mathrm{Pu}$. Gamma-gamma coincidence has been proven capable of significantly enhancing the signal to background ratio, which is advantageous in the characterization of an isotope with a complicated decay scheme, like ${ }^{239} \mathrm{Pu}$

The use of gamma-gamma coincidence is most valuable when used on radionuclides that decay to a short-lived excited state, then immediately decay to a lower energy state. In this situation, the cascade gamma emission from the decays would be in coincidence. For this reason, the use of coincidence has been demonstrated to significantly reduce background counts [5]. The use of gamma-gamma coincidence produces a count reduction of 3 to 4 magnitudes when measuring selenium $\left({ }^{75} \mathrm{Se}\right)$ in environmental standards using neutron activation analysis [6]. Gamma-gamma coincidence counting allows for lower detection limits, particularly in conjunction with the use of energy gating. A comparison of normal and coincidence gated spectra was performed and an improvement in both peak to background ratio and detection limits with the use of selenium was determined [7]. Data has also been produced demonstrating lower detection limits and reduced background counts with the use of gammagamma coincidence for measuring ${ }^{22} \mathrm{Na}$ [8]. Gammagamma coincidence techniques have been used for environmental monitoring of ${ }^{22} \mathrm{Na}$ with bismuth germanate scintillators (BGO) [9]. The Pixie-4 module has been used in conjunction with sodium iodide (NaI) detectors for the detection of enriched and depleted uranium [10]. In addition, the use of coincidence techniques has been demonstrated to be effective in deconvolution of overlapping peaks, particularly in ${ }^{76} \mathrm{As}$, ${ }^{160} \mathrm{~Tb}$, and ${ }^{169} \mathrm{Yb}$ [11]. The goal of this research is to demonstrate the feasibility of gamma-gamma coincidence for identifying specific energy peaks in the coincidence spectrum for medium to high activity levels of $239 \mathrm{Pu}$.

\section{EXPERIMENTAL}

Our gamma-gamma coincidence system at the Nuclear Engineering Teaching Laboratory utilized two high-purity germanium detectors, both of which are p-type. One of the HPGe detectors is a Canberra CG2518 which has a relative efficiency of $25 \%$ and an energy resolution of $1.95 \mathrm{keV}$ full width half maximum (FWHM) at $1332 \mathrm{keV}$ of ${ }^{\circ} \mathrm{Co}$. The second HPGe detector is also a Canberra CG2519 which has a relative efficiency of $15 \%$ and an energy resolution of $1.9 \mathrm{keV}$

*cegozi@utexas.edu 
FWHM at $1332 \mathrm{keV}$. The detectors are linked to a digital XIA Pixie-16 module with digital spectrometry and coincident data acquisition capabilities [12]. When measuring in singles, the data from only one of the two detectors is used. When measuring in coincidence, only gammas that reach both detectors at the same time are recorded in the spectrum. An image of the coincidence setup is shown below in Figure [1].

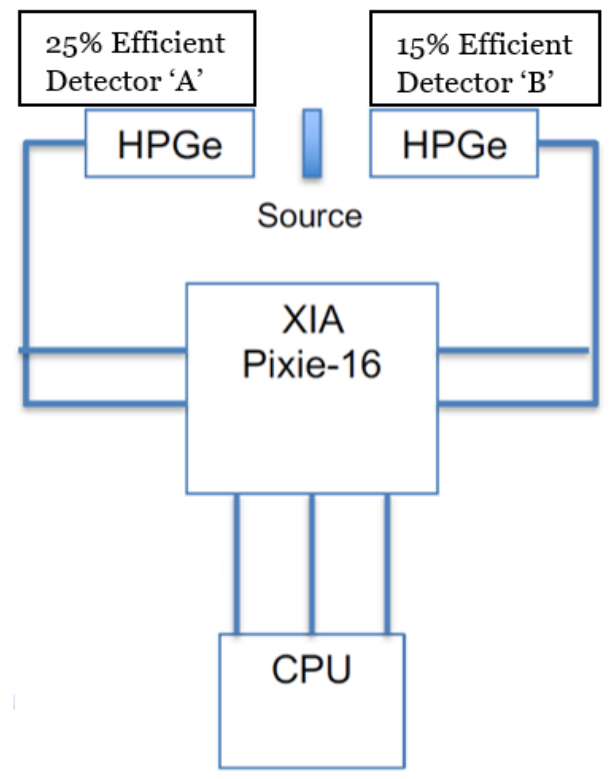

Figure 1. HPGe detectors coincidence setup used for all measurements.

To determine the feasibility of unshielded background counts detected with coincidence compared to singles, a spectrum was taken using both systems with no source for 5 hours as shown in Figure [2].

This comparison proves that the use of gammagamma coincidence significantly reduces the background counts without the use of any shielding.

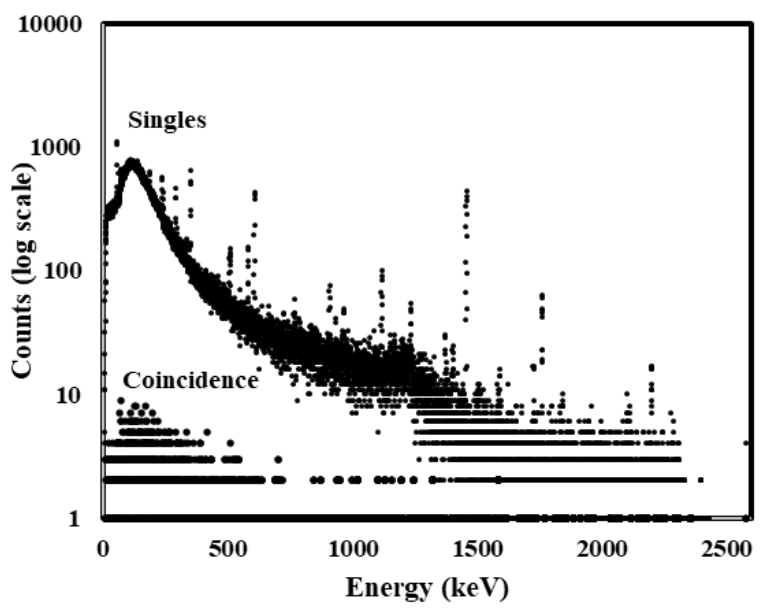

Figure 2. HPGe coincidence and singles background spectra.

A ${ }^{60}$ Co source was measured in both singles and coincidence schemes at various inter-distances between the two end-caps of the detectors, and the $1332 \mathrm{keV}$ net area counts were compared. This was done to show how the counting geometry affects net counts in singles and coincidence. Using only detector ' $A$ ', the net counts decreased by an order of magnitude at a source to detector distance of $10 \mathrm{~cm}$. Using the two detectors in coincidence, however, results in a decrease of two orders of magnitude in the counts at a source to detector distance of $10 \mathrm{~cm}$. The singles and coincidence data at varying distances are displayed below in Figure [3] and Figure [4].

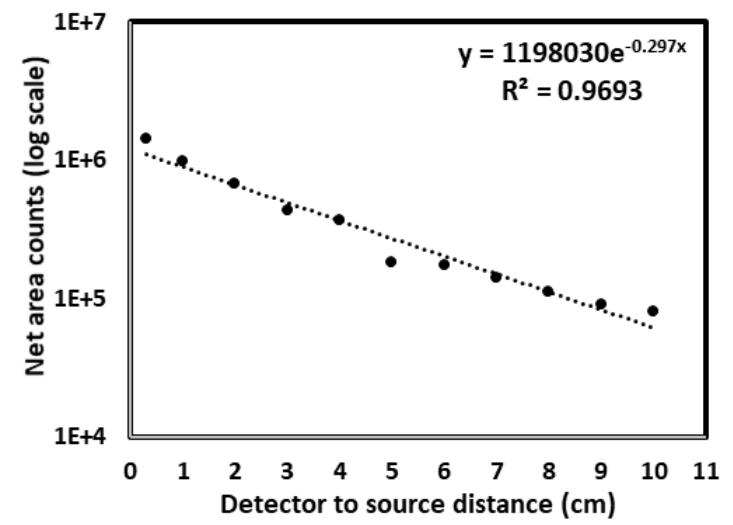

Figure 3. HPGe singles $1332 \mathrm{keV}$ net area counts at increasing equidistant source to detector distances measured for 5 hours.

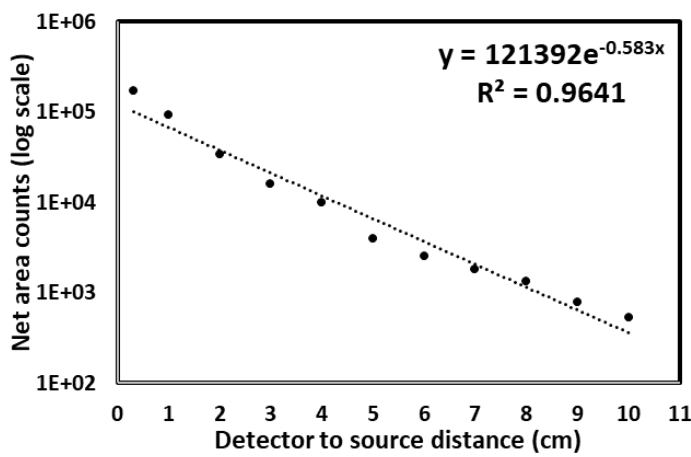

Figure 4. HPGe coincidence ungated $1332 \mathrm{keV}$ net area counts at increasing equidistant source to detector distances measured for 5 hours.

From the figures above, it can be seen that the reduction in counts with the use of coincidence becomes more significant at greater distances. This is to be expected, as the number of gamma rays that reach both detectors within the coincidence window is inversely related to source to detector distance. In this setup, the coincidence window is set to 80 nanoseconds because when the counts are high, the coincidence window needs to be smaller to prevent unwanted counts in the coincidence spectrum. The window of 80 nanoseconds was determined to be the best window after multiple rounds of optimization.

\subsection{Characterization of Pixie-16 System}

After determining the feasibility of the use of gamma-gamma coincidence for increasing peak to background ratio, a characterization of the Pixie-16 system was performed. We verified that the peaks 
acquired with this system follow the Poisson statistics by placing a ${ }^{60} \mathrm{Co}$ source equidistant between the two detectors and performing 2500 runs on the system. Each run was 200 seconds, and a custom Python code that automatically started and stopped each run was used to ensure the elimination of human error. The coincidence data was analyzed from the Pixie- 16 system and the distribution in Figure [5] below was produced. The red dashed line is an overlay of the theoretical Poisson distribution, which is a good fit to the normalized data.

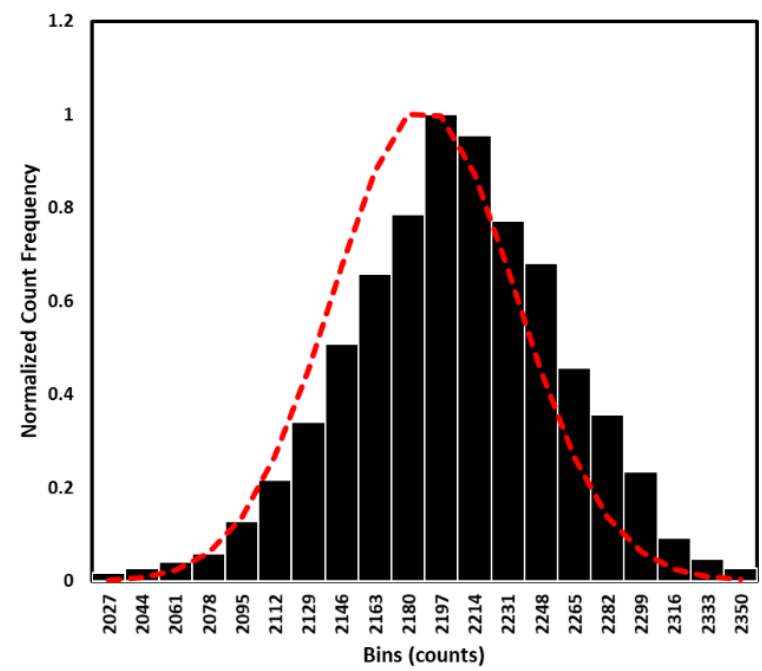

Figure 5. Poisson distribution coincidence HPGe 2500 runs with 200 s counting time for $1332 \mathrm{keV}{ }^{60} \mathrm{Co}$ gamma.

From the dataset generated, the Pixie-16 system abides by Poisson distribution.

\subsection{Advantages of Coincidence Counting for ${ }^{239} \mathrm{Pu}$}

The value in using ${ }^{239} \mathrm{Pu}$ in the gamma-gamma coincidence system can be determined from its decay scheme. A simplified version of the decay scheme of ${ }^{239} \mathrm{Pu}$, which decays by alpha emission to ${ }^{235 \mathrm{U}}$, is shown in Figure [6], with only the usual major gamma rays displayed.

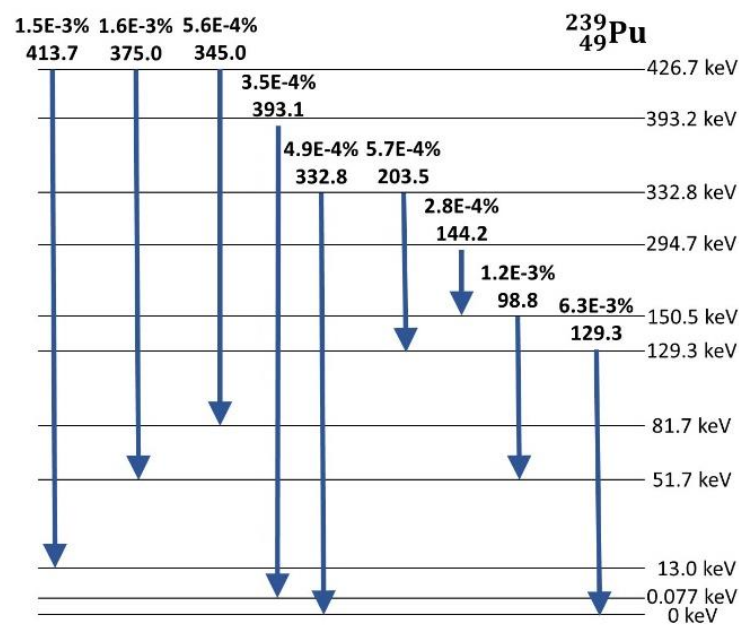

Figure 6. Major gammas belonging to the ${ }^{239} \mathrm{Pu}$ decay scheme [13]
In the decay scheme, there are multiple gamma rays that are in coincidence with each other, including the $144 \mathrm{keV}$ and $98.8 \mathrm{keV}$ gamma rays, and the $203 \mathrm{keV}$ and $129.3 \mathrm{keV}$ gamma rays. Therefore, the use of gammagamma coincidence on ${ }^{239} \mathrm{Pu}$ could be effective due to the isotope's gamma rays that are in coincidence, e.g. in plutonium reprocessing or nuclear security applications

\subsection{Coincidence Gating of ${ }^{239} \mathrm{Pu}$}

Energy gates have been set in the coincidence spectra to further reduce the background radiation. Figure [7] includes an overlay of singles and coincidence spectra of a $2.31 \mathrm{E}-4 \mathrm{~cm}$ thick ${ }^{239} \mathrm{Pu}$ foil electrodeposited on nickel with an activity of $1.81 \mathrm{E} 6 \mathrm{~Bq}$. The ratio of intensity to initial intensity due to self-attenuation of the foil is 0.995 and is therefore negligible.

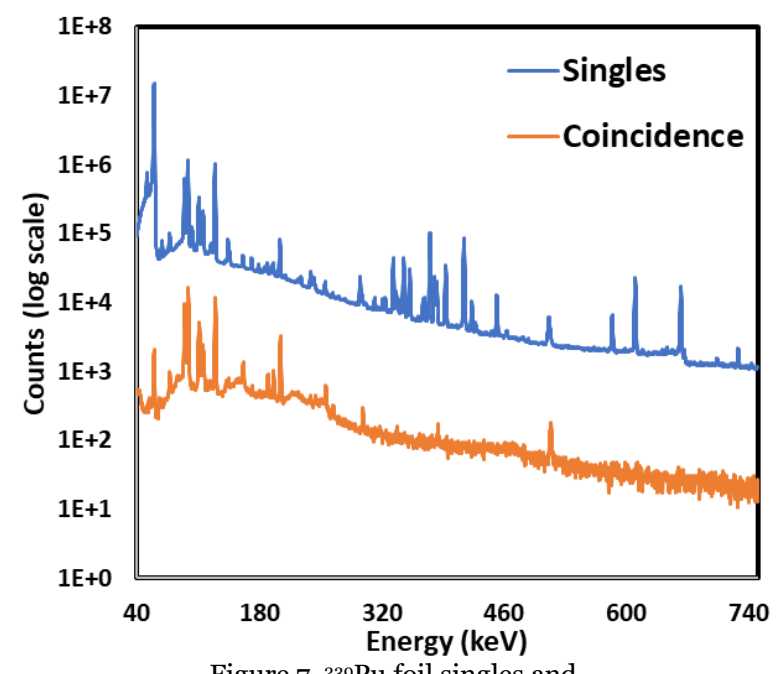

Figure 7. ${ }^{239} \mathrm{Pu}$ foil singles and coincidence with 97-hour live time count.

The comparison of the singles and coincidence data was then used to evaluate the gamma rays in coincidence. From Figure [7], it can be seen that the most gammas in both true and random coincidences are in the range of $94 \mathrm{keV}$ to $203 \mathrm{keV}$. Therefore, several gammas within the range were analyzed further by energy gating.

The gating was done using energy gating software (internally developed), in which the energy of interest at detector 'A' was specified by the user after performing a measurement with the two detectors $0.3 \mathrm{~cm}$ from the ${ }^{239} \mathrm{Pu}$ source. We realize that this is not an ideal geometry due to summing and pileup, however, this research was to evaluate the use of gamma-gamma coincidence, rather than to make activity measurements. The software then found all energy peaks in detector ' $\mathrm{B}$ ' that were in coincidence with the specified energy. This process was performed on the $94 \mathrm{keV}, 103 \mathrm{keV}, 111 \mathrm{keV}$, and the $129 \mathrm{keV}$ energy peaks.

The most successful gated spectrum demonstrates experimentally that the gamma ray at $203 \mathrm{keV}$ is in coincidence with the $129 \mathrm{keV}$ gamma. The other peaks that appear in the gated spectrum are random coincidences. In addition, the gated spectrum has a background count reduction of 3 orders of magnitude 
relative to the coincidence data, and 5 orders of magnitude reduction relative to the singles data as shown in Figure [8].

To quantify the increase in peak to background ratio, a comparison of the counts of the $203 \mathrm{keV}$ peak in the singles, coincidence, and gated spectra was done. The background counts for each of the spectra was determined and a ratio of peak to background counts was calculated. The $203 \mathrm{keV}$ peak is indicated by the red box in Figure [8] below, and the net counts are shown in Table 1.

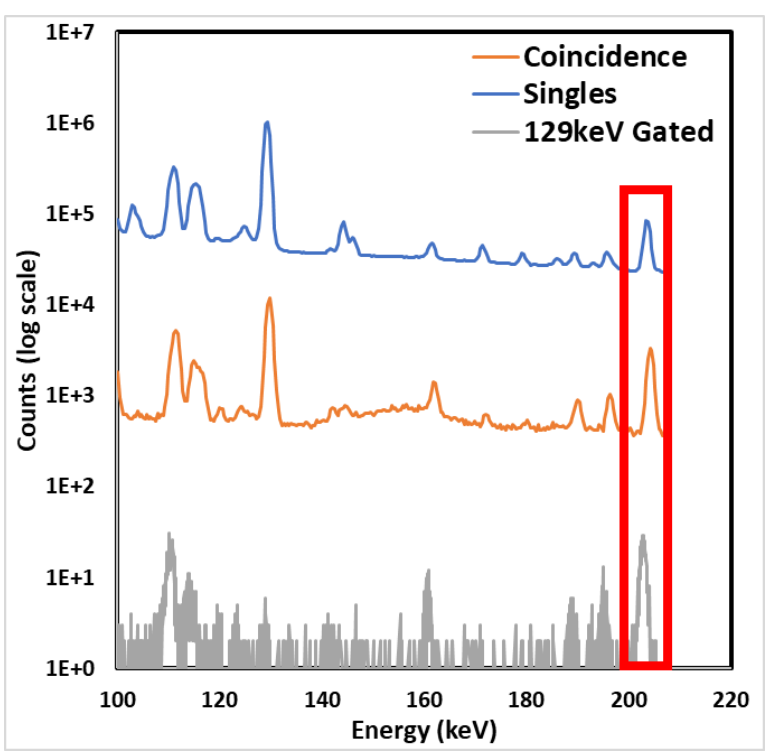

Figure 8. Singles, coincidence, and $129 \mathrm{keV}$ gated spectra comparison of $203 \mathrm{keV}$ peak.

Table 1. Counts in background and $203 \mathrm{keV}$ peak in singles, coincidence, and gated spectra, and peak to background ratio

\begin{tabular}{|l|c|l|l|}
\hline 203 keV Peak & $\begin{array}{l}\text { Background } \\
\text { Counts }\end{array}$ & $\begin{array}{l}\text { Peak } \\
\text { Counts }\end{array}$ & $\begin{array}{l}\text { Peak to } \\
\text { Background Ratio }\end{array}$ \\
\hline Singles & 23678 & 84279 & 3.6 \\
Coincidence & 416 & 3287 & 7.9 \\
129 keV Gated & 2 & 27 & 13.5 \\
\hline
\end{tabular}

The peak to background ratio of the energy gated data is 3.75 times greater than that of the singles ratio, from Table 1. This increase of greater than double the ratio indicates that gamma-gamma coincidence can be used effectively with the absence of shielding.

\section{CONCLUSION}

Based on the reduced background and increased peak to background ratio, gamma-gamma coincidence can be used to identify ${ }^{239} \mathrm{Pu}$ for homeland security, nonproliferation, and nuclear fuel cycle purposes. Gamma-gamma coincidence can be used for ${ }^{239} \mathrm{Pu}$, as the isotope has several gamma rays in coincidence, including the 98, 144, 129, and $203 \mathrm{keV}$ gammas. The use of energy gating in addition to coincidence further reduces the background by 3 more orders of magnitude and is an efficient method to experimentally determine the gamma rays in coincidence with a particular gamma ray.

The lack of shielding required for background reduction, in addition to the numerous gamma rays of the isotope in coincidence make this method ideal for verification purposes of ${ }^{239} \mathrm{Pu}$. At present, gamma-ray spectroscopy is used for determination of plutonium isotopic measurements [14]. However, gamma-gamma coincidence can be used to determine the plutonium isotopic composition more effectively, even in samples with high americium content. This paper demonstrates the feasibility of the use of gamma-gamma coincidence in isolating ${ }^{239} \mathrm{Pu}$ gamma rays for identification.

Acknowledgements: The authors would like to acknowledge that this research done is within the Los Alamos National Laboratory project 421744, $L A-U R-21-29470$.

\section{REFERENCES}

1. R. Gunnink, J. B. Niday, P. D. Siemens, System for plutonium analysis by gamma ray spectrometry, Part I: Techniques for analysis of solutions, Rep. UCRL51577(Pt.1), LLL, Livermore (CA), USA, 1974. https://doi.org/10.2172/4291806

2. Th. E. Sampson, S-T. Hsue, J. L. Parker, S. S. Johnson, D. F. Bowersox, "The determination of plutonium isotopic composition by gamma-ray spectroscopy," Nuc. Inst. Methods in Phys. Research, vol. 193, no. 1-2, pp. $177-183,1982$. https://doi.org/10.1016/0029-554X(82)90693-0

3. S. Hurtado, M. García-León, R. García-Tenorio, "Optimized background reduction in low-level gammaray spectrometry at a surface laboratory," Appl. Rad. Isot., vol. 64, no. 9, pp. 1006-1012, 2006. https://doi.org/10.1016/j.apradiso.2006.01.008

4. N. Marković, P. Roos, S. P. Nielsen, "Digital gammagamma coincidence HPGe system for environmental analysis," Appl. Rad. Isot., vol. 126, pp. 194-196, 2017. https://doi.org/10.1016/i.apradiso.2016.12.017

5. J. A. Cooper, "Radioanalytical applications of gammagamma coincidence techniques with lithium-drifted germanium detectors," Anal. Chem., vol. 43, no. 7, pp. 838-845, 1971. https://doi.org/10.1021/ac60302a017

6. L.E. Wangen, E. S. Gladney, J.W. Starner, W. K. Hensley, "Determination of selenium in environmental standard reference materials by a .gamma.-gamma. coincidence method using lithiumdrifted germanium detectors," Anal. Chem., vol. 52, no. 4, pp. $765-767,1980$ https://doi.org/10.1021/ac50054a037

7. Truong Van, Minh et al., "Determination of selenium in environmental sample by gamma-gamma Coincidence method," in Proc. of $3^{\text {rd }}$ Int. Conf. on Advances in Applied Science and Environmental Engineering (ASEE 2015), Kuala Lumpur, Malaysia, 2015, pp. 67-70. Retrieved from:

https://www.researchgate.net/publication/324967842 Determination of selenium in environmental sam ple by gammagamma Coincidence method Retrieved on: August 1, 2021

8. J. Konki et al., "Comparison of gamma-ray coincidence and low-background gamma-ray singles spectrometry," Appl. Rad. Isot., vol. 70, no. 2, pp. 392-396, 2012. https://doi.org/10.1016/j.apradiso.2011.10.004

9. W. Zhang et al., "A system for low-level the cosmogenic ${ }^{22} \mathrm{Na}$ radionuclide measurement by gamma-gamma 
coincidence method using BGO detectors," J. Radioanal. Nucl. Chem., vol. 287, pp. 551-555, 2011.

http://doi.org/10.1007/s10967-010-0758-3

10. W. Zhang et al., "A gamma-gamma coincidence spectrometric method for rapid characterization of uranium isotopic fingerprints," J. Radioanal. Nucl. Chem., vol. 288, pp. 43-47, 2011

https://doi.org/10.1007/s10967-010-0868-y

11. A. Drescher, M. Yoho, S. Landsberger, "Gamma-Gamma Coincidence in Neutron Activation Analysis," J. Radioanal. Nucl. Chem., vol. 318, pp. 527-532, 2018. https://doi.org/10.1007/s10967-018-6033-8

12. Pixie-16: 16-Channel PXI Digital PULSE Processor for Nuclear Spectroscopy, XIA LLC, Oakland, CA, USA.
Retrieved from: https://xia.com/dgf pixie-16.html Retrieved on: August 1, 2021

13. NuDat version 2, NNDC at Brookhaven National Laboratory, Upton, NY, USA.

Retrieved from:

https://www.nndc.bnl.gov/nudat2/chartNuc.jsp

Retrieved on: August 1, 2021

14. T. E. Sampson, Plutonium isotopic composition by gamma-ray spectroscopy: a review, Rep. LA-10750MS, Los Alamos National Laboratory, Los Alamos, NM, USA, 1986.

https://doi.org/10.2172/5265462 